\title{
Experimental revealing of asynchronous transient-soliton buildup dynamics

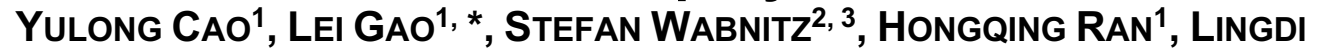 $\mathrm{KONG}^{1}$, AND TAO ZHU ${ }^{1,}$ *
}

${ }^{1}$ Key Laboratory of Optoelectronic Technology \& Systems (Ministry of Education), Chongqing University, Chongqing 400044, China

${ }^{2}$ Dipartimento di Ingegneria dell'Informazione, Elettronica e Telecomunicazioni, Sapienza Università di Roma, Via Eudossiana 18, 00184 Rome, Italy

${ }^{3}$ Novosibirsk State University, 1 Pirogova str, Novosibirsk 630090, Russia

*Corresponding author: gaolei@cqu.edu.cn; zhutao@cqu.edu.cn

\begin{abstract}
The buildup process of coherent structures and patterns from the composite balance between conservative and dissipative effects is a universal phenomenon that occurs in various areas of physics, ranging from quantum mechanics to astrophysics. Dissipative solitons are highly coherent solutions of nonlinear wave equations, and provide an excellent research platform for ultrafast transient phenomena. Here, by taking advantage of the fast detection technique provided by the dispersive Fourier transform, we experimentally observe the spectral broadening and breathing behavior of transient dissipative structures produced asynchronously during the buildup process of dissipative solitons. These observations unveil a novel dynamics of dissipative soliton generation, which is accompanied by energy quantization, self-phase modulation induced spectral broadening, structural dissipative soliton formation, and Raman soliton self-frequency shifting, thus providing a new insight in transient ultrafast laser dynamics.
\end{abstract}

\section{Introduction}

Dissipative solitons (DSs) are universal spatio-temporal localized waveforms, arising from a balance between nonlinearity, dispersion, loss and gain [1-4]. When operating in a stable modelocking regime, DS lasers provide coherent and high-performance sources of ultrafast pulses, which have found extensive applications. Because of their dissipative origin, DS emerge spontaneously from laser self-organization, and exhibit a particle-like nature. DS have been regarded as an excellent platform for exploring nonlinear optical dynamics in many field of physics, ranging from fluid dynamics, plasmas, optical fibers, complex networks, and BoseEinstein condensates [5-9]. So far, many striking soliton dynamics have been investigated in dissipative soliton fiber lasers, including dissipative soliton molecules [10], rogue waves [3,11], dissipative soliton rain [12], dissipative soliton explosions [13] and dissipative soliton resonances [14]. Typically, the dynamics of soliton formation in non-integrable physical systems is non-repetitive, and it exhibits a complex behavior, owing to the energy redistribution among the various internal modes, before a stationary soliton settles down [15]. When compared with the extensive theoretical studies, the experimental observations of soliton formation dynamics are still relatively scarce. Nevertheless, during the buildup dynamics of DSs, the field recirculating in a laser cavity exhibits spectral sharp peaks with oscillating structures [16], as well as the generation of high amplitude waves, that may cause damaging effects to the ultrafast laser. Therefore, the experimental investigation of the buildup dynamics of DSs has a great impact on practical applications, in addition to its important significance for fundamental research.

Capturing ultrafast transient phenomena requires real-time spectral measurements. Thanks to the time-stretch dispersive Fourier transform (TS-DFT) technique [17], the transient dynamics of solitons in fiber lasers have been extensively experimentally investigated in recent years. The TS-DFT technology stems from the spatiotemporal duality, namely, the transmission of a temporal pulse in a dispersive element with a sufficiently large dispersion is analogous to the diffraction of a beam passing through a lens in the far-field approximation. The frequencydomain spectral information of the pulse is thus mapped into the temporal pulse waveform, and 
the stretched light pulse has the same shape as the spectral intensity envelope. Together with high-speed photodetectors (PDs) and real-time oscilloscopes, several important studies have been carried out by different groups. For example, the TS-DFT permitted to study the transient buildup characteristics of nonlinear Schrodinger solitons [18], the process of Kerr lens modelocking in titanium sapphire lasers [19], and distinctive birth and extinction dynamics of three types solitons [20]. In addition, the transient dynamics during the buildup process of DSs have also been studied by the research groups of Dudley [21], Zhichao Luo [16] and Heping Zeng [15], respectively. These works have revealed several different mode-locking mechanismbased striking nonlinear phenomena, including the transient emergence and decay of coherent multi-soliton states, SPM-induced instability, dissipative soliton splitting, spectral oscillation structures, transient soliton molecules in the process of soliton formation. Nevertheless, the complex dissipative nonlinear phenomena in optical fiber ring cavities are far from being fully explored. The entire process of pulses competing evolution and the transient multi-soliton generation during the buildup of the dissipative soliton has still not been discovered. For a complete understanding of the complex transient buildup dynamics of DSs, additional experimental investigations are necessary.

In this work we report, we believe for the first time, on the experimental observation of transient soliton pairs, which are mutually ignited during the buildup process of DSs for pump power larger than that for a stable DS. We observe intermittent spectral structures, alternating between oscillating and stable behavior. At the same time, we observed that each transient soliton is accompanied by a complex evolution process of its spectral broadening, exhibiting oscillating sharp spectral peaks, spectral breathing and wavelength shifting. The obtained results enable us to broaden the horizons of DS buildup dynamics in ultrafast fiber lasers, which will be beneficial for both the nonlinear optics and ultrafast laser communities.

\section{Experimental setup}

Figure 1 schematically depicts our ring fiber cavity, containing a $15 \mathrm{~m}$ erbium-doped fiber (EDF, Nufern, EDFC-980-HP), backward pumped by a $980 \mathrm{~nm}$ continuous wave (CW) laser through a wavelength division multiplexer (WDM), a polarization independent optical isolator (ISO), a polarization controller (PC), $2.7 \mathrm{~m}$ of single mode fiber (SMF), and an optical coupler (OC) with a $10 \%$ output port. The dispersion parameters of the EDF and SMF are -12.2 and 18 $\mathrm{ps} / \mathrm{nm} / \mathrm{km}$, respectively. Therefore, the total $17.8 \mathrm{~m}$ ring cavity possesses a net normal dispersion of $0.171 \mathrm{ps}^{2}$. The saturable absorbing film is made by mixing $10 \mathrm{wt} \%$ aqueous polyvinyl alcohol and $0.5 \mathrm{mg} / \mathrm{mL}$ single-wall-carbon-nanotubes solution at a volume ratio of $1: 2$, then dehydrated in vacuum [22]. Its nonlinear optical response is measured by means of a home-made balanced two-detector. The modulation depth and the saturation power are $4.9 \%$ and $1 \mathrm{~nJ} / \mathrm{cm}^{2}$, respectively [22]. Such low saturation power permits our laser system to operate in a DS regime with relatively low pump powers.

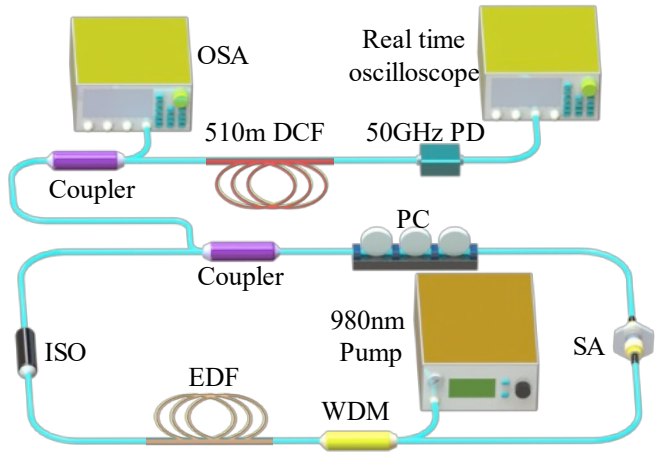

Fig. 1. Schematic of the ring fiber laser cavity and measurement methods. 
The laser output is characterized by an optical spectrum analyzer (OSA, Yokogawa, AQ6370). Meanwhile, we detect the laser single-shot spectra by means of a home-made TSDFT, where periodic signals are stretched by a $510 \mathrm{~m}$ dispersion compensation fiber (DCF) with the dispersion about $-400 \mathrm{ps} / \mathrm{nm} / \mathrm{km}$ for the frequency-to-time transformation, and subsequently fed to a $50 \mathrm{GHz}$ PD connected to a real time oscilloscope (Tektronix, MSO 73304DX) with the bandwidth of $33 \mathrm{GHz}$. The spectral resolution of the TS-DFT system that we built can be calculated according to the formula [17]

$$
\Delta \tau=|D| \cdot \mathrm{L} \cdot \Delta \lambda
$$

where $\Delta \lambda$ is the bandwidth of the spectrum, $\mathrm{D}$ is the group delay dispersion per unit length of the dispersive element (usually in $\mathrm{ps} / \mathrm{nm} / \mathrm{km}$ ), and $\Delta \tau$ is the duration of the spectral mapping. According to the working bandwidth of the oscilloscope, the D value and the length of the DCF, the spectral resolution of the TS-DFT system can be calculated to be $0.15 \mathrm{~nm}$. And the timespectrum conversion scale is $200 \mathrm{ps} / \mathrm{nm}$. Benefiting from the fast response time of the PD, the TS-DFT can record spectra at a scan rate which is remarkably higher than that of conventional space-domain spectrometers. This enables the measurement of round-trip resolved spectra, a technique which has recently been utilized to reveal various fast dynamics in lasers.

Laser operation is obtained at the pump power of $20 \mathrm{~mW}$. Upon further increase of the pump power to $65 \mathrm{~mW}$, we observe a stable DS generation. A typical rectangular temporal profile for the DS was obtained, with a center wavelength of $1569 \mathrm{~nm}$, and the bandwidth of $12 \mathrm{~nm}$. The averaged optical spectrum displayed in $\mathrm{dBm}$-scale is the same as the experimental results of Ref. [23] with the net dispersion of $+0.024 \mathrm{ps}^{2}$. In order to conveniently compare the spectra obtained by the two methods, we display the average spectrum in linear coordinates shown in Fig. 2(a). The pulse duration of DS is 30.5 ps. By utilizing the TS-DFT technique, the singleshot spectrum is displayed on the oscilloscope, as shown in Fig. 2(b). The pulse corresponding to the spectral shape as displayed by the oscilloscope has a duration of 2.4 nanoseconds, which corresponds to the spectral bandwidth of $12 \mathrm{~nm}$ that is obtained by OSA, thus exactly matching the temporal-spectral conversion scale that we estimated. Figure 2(c) shows the spectra over 600 consecutive round-trips during about $78 \mu \mathrm{s}$, which shows that at pump power of $65 \mathrm{~mW}$ the DS laser remains rather stable, and no soliton explosion, multi-soliton or breather is generated.
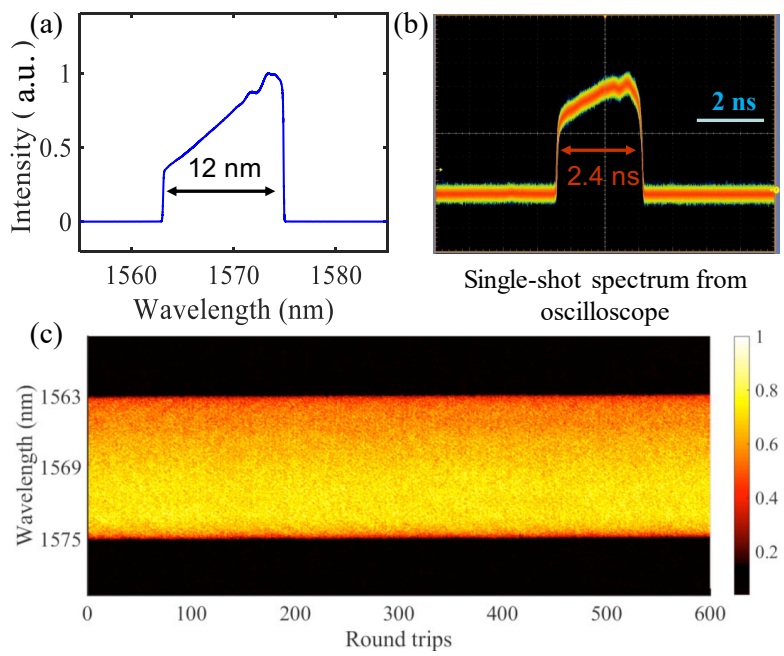

Fig. 2. (a) Averaged optical spectrum obtained by OSA under pump power of $65 \mathrm{~mW}$. (b) Realtime spectrum displayed on oscilloscope. (c) Real-time 2D spectral evolution dynamics of a stable DS. 


\section{Experimental results and discussion}

In order to investigate the DS buildup process in the ultrafast fiber laser, we use a mechanical switch to control the $980 \mathrm{~nm}$ pump laser, with the pump power of $70 \mathrm{~mW}$. We observe the asynchronously triggered transient solitons by skillfully setting the pump power higher than the threshold power required to generate a single soliton and carefully regulating the positions of the PC. A power beyond the laser threshold can provide energy to excite the formation of multisoliton [24]. Results reported in Figs. 3(a)-3(b) illustrate the oscilloscope after transmission in DCF. Fig. 3(a) shows a starting process of DS during 600us about 4500 roundtrips, while the Fig. 3(b) shows another starting process with $5 \mathrm{~ms}$ about 37500 roundtrips. The intensity jitter of these two processes does not occur at the same time, which is consistent with the description in the reference 19 (the buildup of soliton is not repetitive events). However, both of figures show the presence of a bunch of intermittent intensity peaks (or intensity jitter) in the singleshot spectra, extending over a time span of about $100 \mu$ s. This means that the laser needs to go through about 800 round-trips before reaching a short-term stable mode-locked state. The intensity jitter exhibits a structure similar to that observed in Ref. [20] at the beginning of DSs formation. It is worth noting that these intermittent intensity jitters appear randomly, and have similar structural features before reaching a stable mode-locking regime. Subsequently, we look for detailed spectral evolution information by dividing the recorded real-time signal into segments whose duration equals the average cavity round-trip time of $129.5 \mathrm{~ns}$. All intensities are normalized with respect to the maximum value in each detection. The first round-trip is determined by the trigging level of the real-time oscilloscope during DFT detection. 

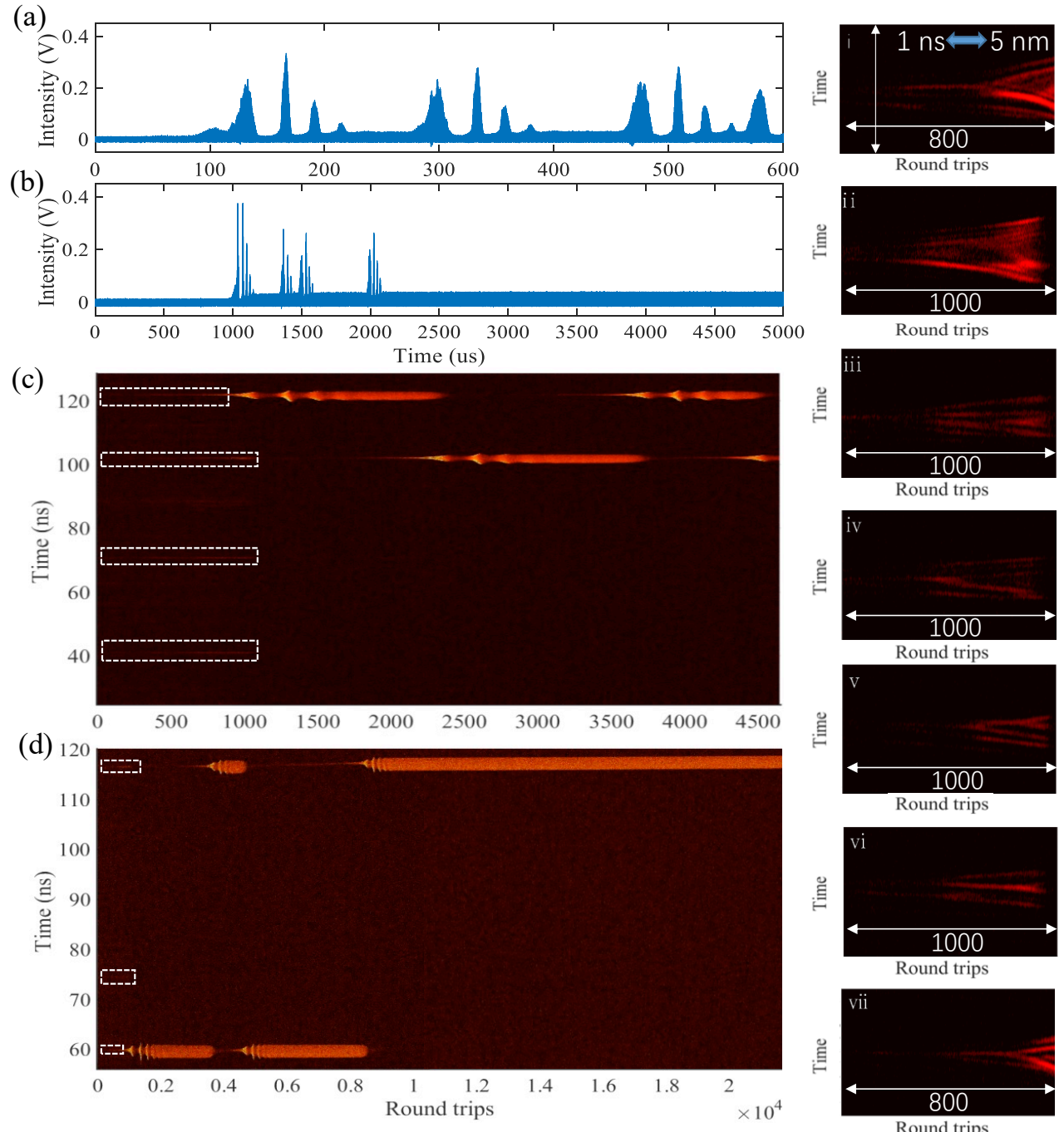

Fig. 3. Real-time observation of two starting processes for DSs at same pump power during (a) 600 us and (b) 5000 us. These are the detection results of the oscilloscope after transmission in the DCF. (c) and (d) illustrate the real-time 2D spectral evolution dynamics of asynchronous transient solitons of 600 us and 5000 us starting processes, respectively. The whited dashed rectangles are the spectral intensity fluctuations. The seven insets are partial enlargements of seven white-dashed rectangles with the range of $1 \mathrm{~ns}(5 \mathrm{~nm})$.

From the 2D real time single-shot spectra in Figs. 3(c)-3(d) we can see that, in the early stage of the laser cavity operation, spectral intensity fluctuations (these are enclosed in the white dashed rectangles) emerge over a few hundred round-trips. The seven insets are partial enlargements of seven white-dashed rectangles with the range of $1 \mathrm{~ns}(5 \mathrm{~nm})$. Figs. i and vii are corresponding to the first surviving and oscillating pulses of Fig. 3(c) and Fig. 3(d). Figs. ii, iii, iv, $v$ and $v i$ are spectral intensity fluctuations, which disappear after spectral broadening. Next, two of these fluctuations in Fig. 3(c) and Fig. 3(d) survive and generate highintensity picosecond pulses that undergo a complex spectral breathing process. We name these pulses as mutually ignited transient solitons (MITSs).

It can be noticed that the spacing between the spectra of the two MITS pulses is different in Fig. 3(c) and in Fig. 3(d), even though these results are obtained for the same pump power. We 
observed that, for a given pump power, the total number of generated MITSs remains the same in every occurrence of a pulse cluster, in agreement with numerical findings [25]. Our laser lasing a stable single dissipative soliton at pump power of $65 \mathrm{~mW}$. When the power is increased to $70 \mathrm{~mW}$, we get such asynchronously triggered transient solitons assisted by a fine setting of the PCs. But below this pump power and at any PC position, we cannot observe the generation of MITSs. The two buildup processes in Figure 3 correspond to the same pump power, but the PC states are different, which is why the timing intervals between the two MITS are different. In other words, the pump power determines whether a single soliton or double transient solitons gradually evolve from the many spectral intensity fluctuations during the initial stage of laser generation. The specific polarization state determines which two spectral intensity fluctuations perform subsequent spectral broadening and breathing oscillation.

The spectra of pulses exhibit an intermittent or oscillating spectral structure. More interestingly, Fig. 3(c) shows that, for a given pump power above the threshold, the transient soliton (MITS-1) emerging around hundreds round-trips, and asynchronously stimulates the generation of a second transient soliton (MITS-2). Next, the MITS-2 pulse undergoes its own stages of spectral broadening and breathing. From Fig. 3(d) we can see that since the pump power is not sufficient to support a stable multi-soliton mode-locking regime, at each point in time only one MITS survives to establish a single-pulse mode-locking regime.

As a matter of fact, the laser is not operating in a true multi-soliton regime. As it can be seen from Fig. 3(d), only one mutually ignited transient soliton (MITS) eventually survives. It is precisely because the energy that we provide is not enough to support the simultaneous oscillation of two solitons, that we observe such a mutual ignition phenomenon. This is the new insight we provide to study the buildup process of DSs. Namely, the buildup process of the final stable single dissipative soliton will also possibly be accompanied by the generation of MITSs, because the pump power is above the threshold. For the generation of such a short transition between the single pulse regime and the multi-pulse regime in the case of a strong net-normal dispersion regime, the employed saturable absorber is vital. The saturable absorber we chosen is SWCNTs, due to their sub-picosecond recovery time and high modulation depth. We have used the same SA to generate DS before [26, 27]. In this case, we additionally use the $\mathrm{PC}$ to help us to observe the mutually ignited soliton dynamics.

In Figs. 4(a)-4(b), we zoom the fast temporal variable to show fine details of the two MITSs. As can be seen, during every oscillation of MITS-1, the single-shot spectrum shows several stages of spectral broadening and breathing, which are different from the buildup dynamics of DSs as reported in Ref $[16,20]$. Here the evolution of the MITS spectrum undergoes a complex evolution: in addition to spectral broadening, the spectrum displays a strong breathing behavior. We may identify following three processes in the evolution of transient solitons. The first stage is the weak broadening of the picosecond pulse, as shown in white dashed circles in Fig. 4(a). Next, the pulse broadens sharply, and its bandwidth breathes with the period of about 200 round-trips, as indicated by the green dashed circles in Fig. 4(a). In the third stage, the spectral shape remains indefinitely upon successive round-trips.

The seven insets in Fig. 4 illustrate the spectrum evolution for typical round-trips, corresponding to the white, green and yellow dashed circles in Fig. 4(a). The first two figures $\mathrm{i}$ and ii show the initial spectral broadening of two MITSs. As can be seen from these figures, the spectrum of the picosecond pulse is gradually broadening and new intensity peaks are generated internally. The frequencies of these peaks gradually approach the two edges of the broadening pulse spectrum (as shown by the white translucent trajectories). Such frequency broadening and multi-peak evolution of the spectrum of pulses are consistent with the picture of self-phase modulation (SPM)-induced spectral broadening. This is because of the time dependent frequency chirp induced by SPM across the pulse. Therefore, different points in time across the pulse will have the same instantaneous frequency. In the corresponding spectrum, the superposition of waves with the same frequency and different phases produce constructive and destructive interferences, resulting in a multi-peak pulse spectrum. The number of peaks is 
determined by the total nonlinear phase shift experienced by the pulse. When the pulse is continuously amplified in the cavity, the nonlinear phase shift will increase with the accumulated fiber length and pulse intensity. Eventually, more frequencies exhibiting interference peaks are generated inside the spectrum, resulting in spectrum broadening. These peaks gradually move towards the edge of the pulse to form a trace as the number of the roundtrips increase. The initial broadening of these spectra will similarly occur in Kolmogorov's cascade resembling the turbulent structures [28]. This structure is also internally created with the appearance of bright and dark stripes due to the formation of incoherence spectral (bright, gray, and dark) solitons [29] appear. Such a phenomenon during the initial stage of dissipative soliton formation universally occurs in both coherent and incoherent systems. For the further stage, the localized structure has a large chirp with no a perfect phase-locking can be interpreted as a partially coherent multi-soliton complex containing a set of nonlinearly coupled internal "modes" localized within a "self-potential" [30]. The "thermalization" of this partially coherent complex can lead to either its splitting/dissolution or formation of single dissipative soliton. 

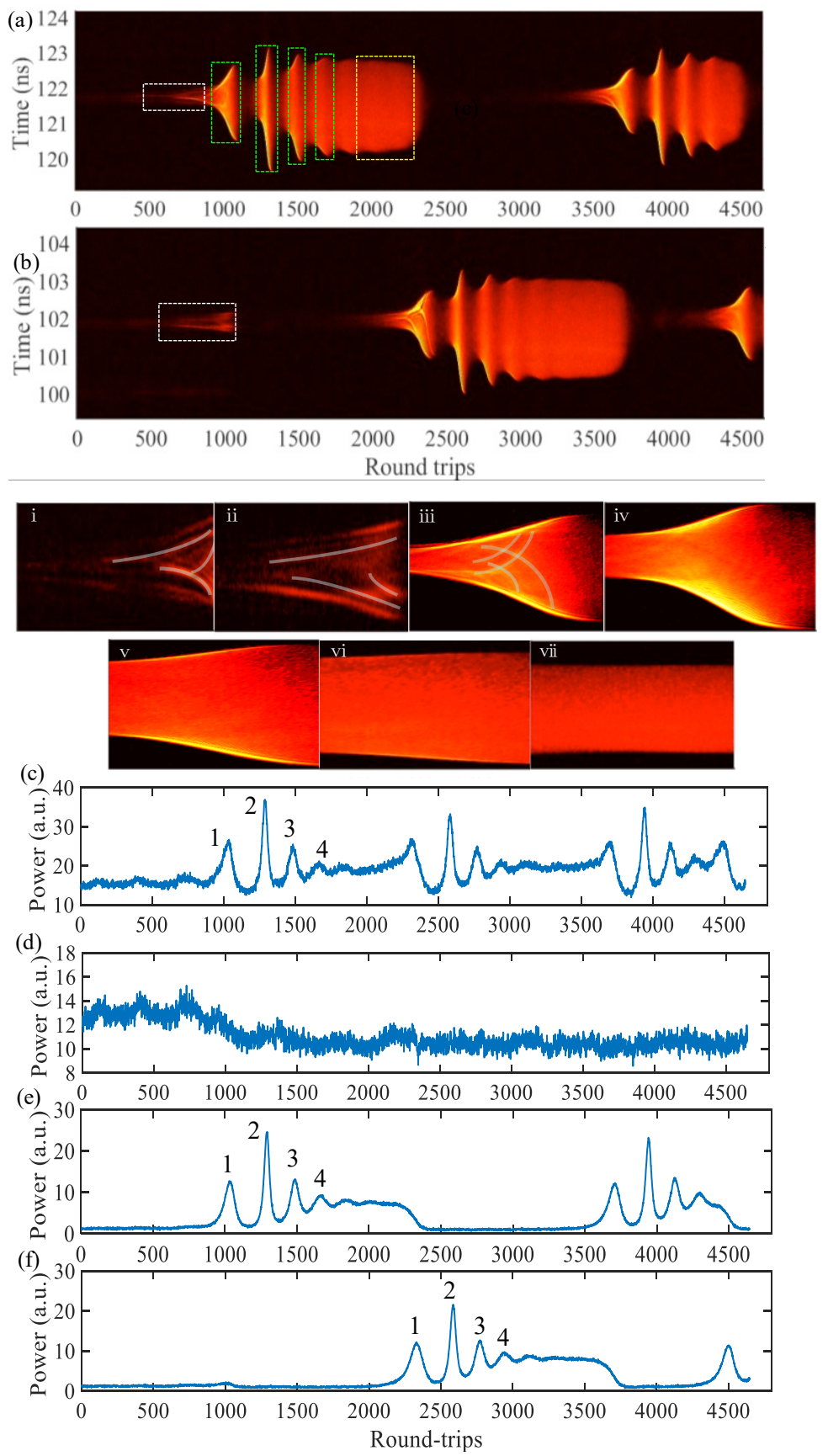

Fig. 4. (a) Real-time 2D spectral evolution dynamics of MITS-1. (b) Real-time 2D spectral evolution dynamics of MITS-2. The seven insets are partial enlargements of white-dashed, green-dashed and yellow-dashed rectangles ( $\mathrm{i}$, ii corresponding to white-dashed rectangles; iii, iv, $v$ and vi corresponding to green-dashed rectangles; vii corresponding to yellow-dashed rectangles.). The white translucent trajectories in $\mathrm{i}$ and $\mathrm{ii}$ are new intensity peaks moving trace. (c) Energy evolution vs. round-trip number. (d) Energy evolution of the background noise with pulses 1 and 2 removed. (e) Energy evolution of MITS-1 with the background noise removed. (f) Energy evolution of MITS-2 with the background noise removed. 
The inserts iii, iv, $v$ and vi in Fig. 4 present partial enlargements of the green dashed area in Fig. 4(a). During the second oscillation of the evolution of transient solitons, the SPMinduced shifting of the multiple spectral peaks is only present in the initial stage of spectral broadening, as shown in the inset iii of Fig. 4. As can be seen, this process leads to a sudden growth of spectral intensity at the edges of the pulse spectrum, where a complex multi-peak structure appears. As the spectral evolution of the pulse progresses, these multiple peaks gradually move away from the spectrum edges, until they disappear within the SPM-broadened spectrum. The appearance highly structured spectral spikes that spread across both edges of spectrum was earlier expounded [31] and experimentally observed [16] known as "structural dissipative soliton". However, the observation of the motion of the spectral spikes (shown in edges of spectra illustrated in insets iii, iv, $v$ of Fig. 4) is a property that was not, to our knowledge, reported before in experiment.

Two typical examples of single-shot spectra, appearing in the second breather of Fig. 4(a), are shown in Fig. 5. Clear moving trajectories of spectral spikes are also visible at the spectral edges of spectra illustrated in insets iii, iv and $v$ of Fig. 4. All because of these spectral structures identify the formation of transient structural dissipative solitons, associated with the pulse shaping mechanism in the normal dispersion regime. In other words, the formation of transient structural solitons leads to high intensity spectral interference patterns to appear at the edge of the mode-locked spectrum. Meanwhile, the pulse shaping mechanism in the normal dispersion regime tends to cut off the wings of the spectrum, thus limiting the energy conversion to the new frequency components generated by SPM and cascaded four-wave mixing (FWM) away from the central wavelength. Under the formation mechanism of DSs, a sharp drop intensity distribution is formed at the edge of the spectrum. Subsequently, as shown by Fig. 5, this means that the multi-peak structure of the spectral edges appears to move inward and gradually disappear.

Finally, the third phase in the evolution of a MITS is shown in the inset vii of Fig. 4: the consecutive single-shot spectra of MITS-1 reach a short-term stable state, with a typical rectangular shape for the spectrum of the DSs.

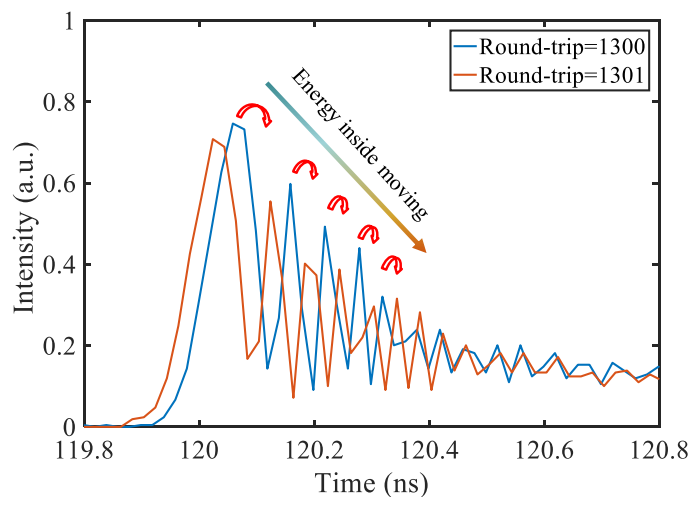

Fig. 5. Motion of spectral spikes moving during spectral broadening.

To gain more insight in the behavior of the mutual ignition among two transient solitons, we investigated the characteristics of the energy evolution during the soliton buildup process. Firstly, we study the evolution of the total intra-cavity energy, by integrating the normalized real-time 2D spectra, and calculating the result for ever round-trip, as shown in Figure 4(c). Before reaching a phase of short-term stabilization, the energy undergoes four damped oscillations (marked with 1, 2, 3, 4 in Fig. 4), corresponding to the formation of transient structural dissipative solitons, exhibiting both spectral broadening and breathing. Particularly, the amplitude of the second spectral intensity peak is especially strong, which is consistent with 
the in-phase superposition of all longitudinal modes. In Fig. 4(d) we display the energy evolution of the background noise with pulses 1 and 2 removed. As we can see, the picosecond scale noisy fluctuations disappear after about 1000 round-trips. The vanishing of the background pulse confirms winner-takes-all law in DS laser systems, where energy is ultimately fed to mode-locking of solitons.

Next, we computed the energy of the two MITS pulses separately in Figs. 4(e)-4(f). Before reaching a short-term stabilization, MITS experience about 750 round-trips of energy fluctuations. In addition, Fig. 4(e) and 4(f) show that a perfect energy exchange occurs among the two pulses: when a pulse is alive and breathing, the energy of the other pulse is almost vanishing. Only when the first soliton reaches a stable state, then energy starts to flow into the second soliton. This is because the energy provided by the pump allows the laser cavity to operate in-between the multi-soliton and single-soliton mode-locking regimes, hence it cannot sustain two simultaneous solitons. As a result, energy is transferred across among the two pulses in a quantized form [25], leading to the formation of transient solitons that experience a mutual ignition. Moreover, the complete energy transfer among MITSs proves that energy is discontinuously exchanged among pulses in such a laser cavity, operating at the edge between the single-soliton and multi-soliton emission regimes.
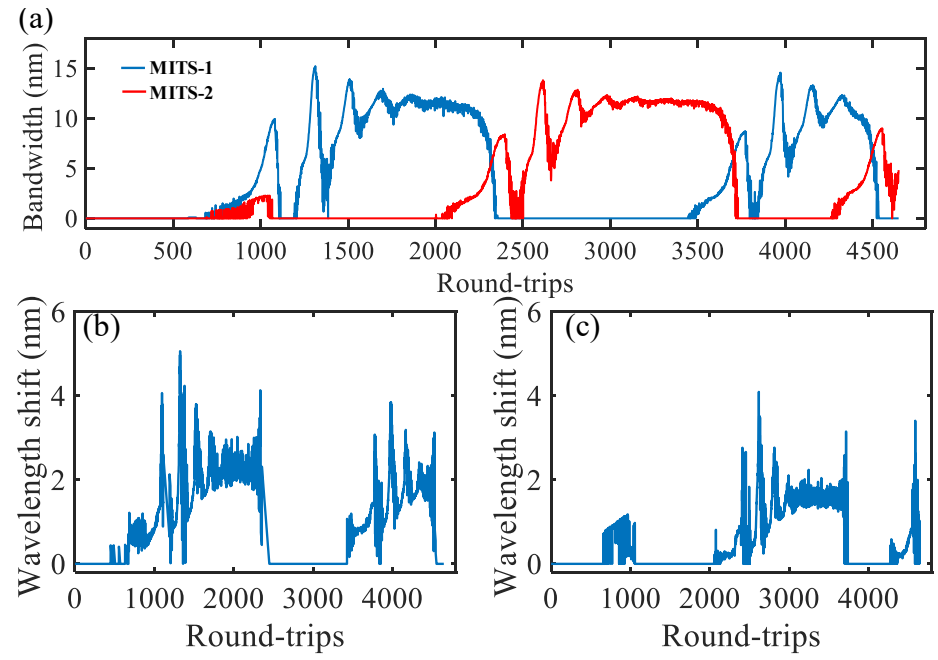

Fig. 6. (a) The bandwidth evolution of MITS 1 and 2. (b) The center wavelength evolution of MITS-1. (c) The center wavelength evolution of MITS-2.

We also calculated the evolution of the $3 \mathrm{~dB}$ bandwidth of each MITS pulse by using the time-spectrum conversion scale of $200 \mathrm{ps} / \mathrm{nm}$, as shown in Fig. 6(a). The blue curve refers to MITS-1, and the red curve refers to MITS-2, respectively. It is worth to note that the period of bandwidth oscillation of the two MITSs is of about 200 cavity round-trips. Soliton bandwidth oscillation is caused by the competition between gain, loss, dispersion and nonlinearity, until a stable DS mode locking state is achieved. After about 1000 periods of oscillation, the bandwidth of each MITS reaches a metastable state, with a bandwidth of about $12 \mathrm{~nm}$, which is the same value as that measured for the steady-state DS. In addition, by carefully observing the spectral evolution of each MITS in Fig. 4(a) and Fig. 4(b), we can see that the soliton spectra remain asymmetrical during the successive broadening and breathing oscillations. The degree of such asymmetry may be quantified by evaluating the evolution of their center-of-mass wavelength. From Figs. 6(b)-6(c), we can see that during the evolution of each MITS their central wavelength is gradually red-shifted. Note that the central wavelength shift qualitatively follows the corresponding energy evolution in Figs. 4(e) and 4(f). Such a power-dependent wavelength shift could be explained as a Raman-induced soliton self-frequency shift [32]. 


\section{Conclusion}

In this work, we experimentally investigated a novel generation dynamics of dissipative solitons in an ultrafast fiber laser with net-normal dispersion. We observed that, for a pump power leading to operation at the edge between multi-soliton and single-soliton mode-locking regimes, mutually ignited transient solitons are generated, fully exchanging their energy in a quantized manner along their propagation. The generation dynamics of these transient solitons is accompanied by self-phase modulation induced spectral broadening. Meanwhile, the formation of transient structural dissipative soliton leads to the generation of oscillation and breathing spectral peaks accompanied with Raman soliton self-frequency shift. The real-time analysis of the generation of mutually ignited transient solitons paves the way for the study of complex dissipative soliton dynamics in nonlinear optical systems fiber lasers.

\section{Funding}

Natural Science Foundation of China (61635004, 61705023), Project supported by graduate scientific research and innovation foundation of Chongqing, China (CYB19037), National Postdoctoral Program for Innovative Talents (BX201600200), Postdoctoral Science Foundation of China (2017M610589), National Science Fund for Distinguished Young Scholars (61825501), Ministry of Education and Science of the Russian Federation (14. Y26.31.0017).

\section{Competing interests}

The authors declare no conflicts of interest

\section{Reference}

1. P. Grelu and N. Akhmediev, "Dissipative solitons for mode-locked lasers," Nat. Photonics 6, 84-92 (2012).

2. J. M. Dudley, F. Dias, M. Erkintalo, G. Genty, "Instabilities, breathers and ogue waves in optics," Nat. Photonics 8, 755-764 (2014).

3. C. Lecaplain, P. Grelu, J. M. Soto-Crespo, N. Akhmediev, "Dissipative rogue waves generated by chaotic pulse bunching in a mode-locked laser," Phys. Rev. Lett 108, 233901 (2012).

4. W. H. Renninger, A. Chong, and F. W. Wise, "Dissipative solitons in normal-dispersion fiber lasers," Phys. Rev. A 77, 023814 (2008).

5. L. F. Mollenauer, R. H. Stolen, and J. P. Gordon, "Experimental observation of picosecond pulse narrowing and solitons in optical fibers," Phys. Rev. Lett. 45, 1095-1098 (1980).

6. S. K. Turitsyn, B. G. Bale, and M. P. Fedoruk, "Dispersion-managed solitons in fibre systems and lasers," Phys. Rep. 521, 135-203 (2012).

7. J. H. V. Nguyen, D. Luo, and R. G. Hulet, "Formation of matter-wave soliton trains by modulational instability," Science 356, 422-426 (2017)

8. E. Kuznetsov, A. Rubenchik, and V. E. Zakharov, "Soliton stability in plasmas and hydrodynamics," Phys. Rep. 142, 103-165 (1986).

9. G. I. Stegeman, and M. Segev, "Optical spatial solitons and their interactions: universality and diversity," Science 286, 1518-1523 (1999).

10. A. Zavyalov, R. Iliew, O. Egorov, and F. Lederer, "Dissipative soliton molecules with independently evolving or flipping phases in mode- locked fiber lasers," Phys. Rev. A 80, 043829 (2009).

11. M. Liu, A. P. Luo, W. C. Xu, and Z. C. Luo, "Dissipative rogue waves induced by soliton explosions in an ultrafast fiber laser," Opt. Lett. 41, 3912-3915 (2016).

12. S. Chouli and P. Grelu, "Rains of solitons in a fiber laser," Opt. Express 17, 11776-11781 (2009).

13. A. F. J. Runge, N. G. R. Broderick, and M. Erkintalo, "Observation of soliton explosions in a passively modelocked fiber laser," Optica 2, 36 (2015).

14. W. Chang, A. Ankiewicz, J. M. Soto-Crespo, and N. Akhmediev, "Dissipative soliton resonances," Phys. Rev. A 78, 023830 (2008).

15. J. S. Peng, M. Sorokina, S. Sugavanam, N. Tarasov, D. V. Churkin, S. K. Turitsyn and H. P. Zeng, "Real-time observation of dissipative soliton formation in nonlinear polarization rotation mode-locked fiber lasers," Communications Physics 1, 1-8 (2018).

16. H. J. Chen, M. Liu, J. Yao, S. Hu, J. B. He, A. P. Luo, W. C. Xu, and Z. C. Luo, "Buildup dynamics of dissipative soliton in an ultrafast fiber laser with net-normal dispersion" Opt. Express 26, 2972-2982 (2018).

17. K. Goda, K. K. Tsia, and B. Jalali, "Serial time-encoded amplified imaging for real-time observation of fast dynamic phenomena," Nature 458, 1145-1149 (2009). 
18. X. Wei, C. Zhang, B. Li, and K. K. Y. Wong, "Observing the spectral dynamics of a mode-locked laser with ultrafast parametric spectro-temporal analyzer." in Conference on Lasers and Electro-Optics, OSA Technical Digest (Optical Society of America, 2015), paper STh3L.4.

19. G. Herink, B. Jalali, C. Ropers and D. R. Solli, "Resolving the build-up of femtosecond mode-locking with singleshot spectroscopy at $90 \mathrm{MHz}$ frame rate," Nat. Photonics 10, 321-326 (2016).

20. Y. D. Cui and X. M. Liu, "Revelation of the birth and extinction dynamics of solitons in SWNT-mode-locked fiber lasers," Photonics Research 7, 423-430 (2019).

21. P. Ryczkowski, M. Narhi, C. Billet, Merolla, JM. Merolla, G. Genty, JM. Dudley, "Real-time full-field characterization of transient dissipative soliton dynamics in a mode-locked laser," Nature photonics 12, 4 (2018).

22. Y. Cao, L. Gao, Y. Li, J. Zhang, F. Li, T. Zhu, "Graphene-based all-optical multi-parameter regulations for an ultrafast fiber laser," Optics letters 43, 18 (2018).

23. J. Wang, Z. Cai, P. Xu, G. Du, F. Wang, S. Ruan, Z. Sun, and T. Hasan, "Pulse dynamics in carbon nanotube mode-locked fiber lasers near zero cavity dispersion," Optics Express 23(8), 19947 (2015)

24. Y. Yu, B. Li, X. Wei, Y. Xu, K. K. M. Tsia, and K. K. Y. Wong, "Spectral-temporal dynamics of multipulse mode-locking," Applied physics letters 110, 201107 (2017).

25. D. Y. Tang, L. M. Zhao, B. Zhao, and A. Q. Liu, "Mechanism of multisoliton formation and soliton energy quantization in passively mode-locked fiber lasers," Phys. Rev. A 72, 043816 (2005).

26. L. Gao, Y. Cao, S. Wabnitz, H. Ran, L. Kong, Y. Li, W. Huang, L. Huang, D. Feng, and T. Zhu, "Polarization evolution dynamics of dissipative soliton fiber laser" Photon. Res., 7(11), 1331-1339 (2019).

27. L. Gao, L. Kong, Y. Cao, S. Wabnitz, H. Ran, Y. Li, W. Huang, L. Huang, M. Liu, T. Zhu, “Optical polarization rogue waves from supercontinuum generation in zero dispersion fiber pumped by dissipative soliton," Optics Express 27(17), 23830 (2019).

28. P. A. Robinson, "Nonlinear wave collapse and strong turbulence," Rev. Mod. Phys. 69, 507 (1997).

29. A. Picozzi, S. Pitois, and G. Millot, "Spectral Incoherent Solitons: A Localized Soliton Behavior in the Frequency Domain," Phys. Rev. Lett. 101, 093901 (2008)

30. N. Akhmediev, W. Królikowski, and A. W. Snyder, "Partially Coherent Solitons of Variable Shape," Phys. Rev. Lett. 81, 4632 (1998).

31. A. Komarov and F. Sanchez, "Structural dissipative solitons in passive mode-locked fiber lasers," Physical review E 77, 066201 (2008).

32. V. L. Kalashnikov, E. Sorokin and I. T. Sorokina, "Mechanisms of spectral shift in ultrashort-pulse laser oscillators," J. Opt. Soc. Am. B 18, 1732-1741 (2001). 